\title{
Evaluation of melting process of the permafrost on Mars: Its implication for surface features
}

\author{
Yoshiko Ogawa \\ Earthquake Research Institute, University of Tokyo, Tokyo, Japan \\ Yasuko Yamagishi \\ Institute for Frontier Research on Earth Evolution, Japan Marine Science and Technology Center, Yokosuka, Japan
}

Kei Kurita

Earthquake Research Institute, University of Tokyo, Tokyo, Japan

Received 20 February 2002; revised 23 July 2002; accepted 16 December 2002; published 30 April 2003.

[1] For supplying massive liquid water to the outflow channels, igneous melting of the permafrost layer could have played a significant role. We numerically simulate the melting process of the permafrost layer induced by magmatic intrusion. The point of our simulation is incorporation of thermal convection in porous media, which has not been modeled well in previous studies of the melting of the permafrost. Our results show that convection in the melted zone causes drastic change in heat transfer, which results in focusing in the growth of the melt region and enhancement of water generation. The resulting melt zone extends vertically up just next to the surface, like a plume with a single column (mushroom structure). The volume of meltwater is considerably more than that expected in the conduction case. These characteristics suggest that a substantial amount of water may exist very near the surface. In response to compaction, segregated liquid water may have erupted out of the ground to form the fluvial features. Such an event would certainly be accompanied by surface destruction, which we can see as chaotic terrain. We propose a consistent scenario of forming surface features around the outflow

channels. INDEX TERMS: 6225 Planetology: Solar System Objects: Mars; 8424 Volcanology:

Hydrothermal systems (8135); 1884 Hydrology: Water supply; 5480 Planetary Sciences: Volcanism (8450); 1823 Hydrology: Frozen ground; KEYWORDS: Mars, permafrost, hydrothermal system, thermal convection, chaotic terrain, outflow channel

Citation: Ogawa, Y., Y. Yamagishi, and K. Kurita, Evaluation of melting process of the permafrost on Mars: Its implication for surface features, J. Geophys. Res., 108(E4), 8046, doi:10.1029/2002JE001886, 2003.

\section{Introduction}

[2] On Mars many traces of fluvial-like features have been identified such as outflow channels, valley networks and so on. They potentially suggest the existence of a substantial amount of liquid water very close to the Martian surface at their formation times. The outflow channels in particular, have the characteristics unique to water erosion and clearly indicate the surface runoff of huge amount of water [Baker and Milton, 1974; Baker, 1982].

[3] Up to now the following two ideas have been proposed to explain the formation of the outflow channels; one is massive release of confined aquifer and another is melting of the ground ice (permafrost).

\subsection{Release of Confined Aquifer}

[4] The potential origin of the outflow channels by the release of water from an aquifer confined beneath the

Copyright 2003 by the American Geophysical Union. 0148-0227/03/2002JE001886\$09.00
Martian permafrost was originally proposed by Carr [1979]. Supposing impacts, faulting, or another catastrophic event as the trigger, any groundwater that is stored in the aquifer should be released through the permafrost layer to the surface. Recently, Max and Clifford [2001] proposed a new trigger, the degassing of gas hydrate. A potential difficulty of the confined aquifer model is that the total volume of water stored in the aquifer may be insufficient to form an outflow channel. The extent of such an aquifer will be limited at its bottom by the self-compaction depth and at its top by the base of the permafrost layer. However, depending on the horizontal expanse of the aquifer, this idea may still remain a viable alternative to the melting of ground ice, discussed below.

\subsection{Melting of the Ground Ice in the Permafrost Layer}

[5] Here we consider the possibility that the volume of water that carved the outflow channels was formed by the melting of ground ice from the heat associated with a magmatic intrusion. Geomorphological evidence indicates an almost ubiquitous existence of the permafrost layer 
[Squyres, 1979; Squyres and Carr, 1986; Carr, 1996]. Therefore, it seems reasonable that a considerable amount of ground ice will melt when an igneous body intrudes beneath the permafrost layer. The process could supply adequate liquid water to result in the fluvial erosion of the surface, evidence of which we observe today as the outflow channels. The greatest challenge for this idea is the timescale for the formation of the meltwater. For example, the rate of producing water might be far less than that required for the formation of the outflow channels $\left(\sim 10^{7-9} \mathrm{~m}^{3} / \mathrm{s}\right.$ at its maximum by Baker [1982], Komar [1979], Robinson and Tanaka [1990]) although the volume necessary to produce the observed erosion may not have been estimated correctly so far. However, collecting the water to form a lake on the surface and resultant collapse of the reservoir would easily enable the expected huge flood event. Valles Marineris and its associated chasmata could have hosted lakes [McCauley, 1978]. Lucchitta et al. [1994] examined the canyon system and cited evidence of the former the existence of liquid water within the chasmata.

[6] In this study, we will explore a physically consistent scenario of the formation of water based on melting of ground ice by magmatic intrusion. This critically depends on the thickness of the permafrost layer. Rossbacher and Judson [1981] estimated a large amount of water $(\sim 7.9 \times$ $10^{6} \mathrm{~km}^{3}$ ) should be stored in the crust as a permafrost layer based on the water budget balance. Clifford [1993] estimated the possible thickness of the permafrost layer by calculation of temperature distribution in the crust. The model parameters he considered are the surface temperature, heat flux, and thermal conductivity of the constituent material. He showed that at the equatorial region $\left( \pm 20^{\circ}\right)$, where outflow channels concentrate the most, the thickness must be $2.27-2.47 \mathrm{~km}$ in nominal value, 0 at its minimum, and $11.0-11.6 \mathrm{~km}$ at its maximum.

[7] Fluvial features, such as outflow channels, are located at periphery regions close to the Tharsis volcanoes, which is our main reason for assuming the igneous melting of permafrost is their most likely origin. Outflow channels are observed in four main areas; Chryse-Acidalia basin, Elysium Planitia, Hellas basin and Amazonis Planitia [Carr, 1996]. We see, without exception, that volcanoes exist near the area where outflow channels initiate. For example the Chryse-Acidalia area, where many outflow channels occur, is next to the Tharsis region, the largest volcanic area on Mars. This strongly suggests water supplied to the outflow channels has been formed by volcano/ice interactions.

[8] Previous investigations on this aspect are quite limited. Only a few studies [Gulick, 1998; McKenzie and Nimmo, 1999] have given quantitative estimates about the melting of ground ice. Gulick [1998] investigated groundwater circulation associated with the presence of a magma chamber. Taking the average of the resultant flow of groundwater, she also estimated the possible contribution to the melting of ground ice. McKenzie and Nimmo [1999] solved numerically the melting process by igneous dyke intrusion. They considered that conductive heat from the magmatic intrusion causes melting of ground ice. Their result shows that the melting zone never reaches the top of the frozen layer. Instead, it is formed as a laterally extended shape at the bottom. Without vertical extension of the melted zone, they supposed that faulting opens a route to introduce water through the permafrost to the surface. Alternatively, they consider the possibility that overpressured melt caused the failure. We consider that thermal convection in the melted zone should have played a critical role on the melting process of the permafrost layer because the effective heat transport by convection is expected to enhance the melting process further. This should provide a quite different scenario for the melting process.

[9] In this paper, we report numerical results of the melting of ground ice in the permafrost layer by magmatic intrusion into an initially frozen porous medium. We particularly focus on two different aspects of melting; one is timescale for melting, enhanced by hydrothermal convection, and the second is the resulting geometry of the melted region. The first aspect is important in the accurate estimate of the meltwater production rate to compare with the flow rate estimated from the observed surface features. The second aspect is quite essential in understanding the potential of the meltwater to the surface. In both respects, our model differs from those of previous investigators who have considered the geothermal melting of ground ice.

[10] This paper is organized as follows. In section 2, the numerical method is shown. In section 3, we demonstrate that convection in the melted zone in the permafrost layer causes a drastic change in the heat transfer system, which results in focusing of the melt region and strong enhancement of water generation. In section 4 , the meltwater development of collapse and fluvial features is discussed in connection with the geothermal melting process studied here.

\section{Numerical Method}

[11] Here we calculate the amount of water produced by the melting of the ice-filled pore space in the martian permafrost layer, as induced by a magmatic intrusion. The key in our modeling is consideration of the potential for thermal convection and heat transfer in the melted zone.

\subsection{Modeling}

[12] We summarize our modeling in Figure 1. A boxshaped region represents the assumed permafrost layer. The permafrost is modeled as a porous medium in which the matrix is rock and the pores are filled with water ice. The thickness is assumed to be several kilometers or less based on Clifford [1993].

[13] We assume that the temperature of the surface is constant at 200 Kelvin and the bottom is basically the same. When magma intrudes in the thick Martian crust, sills (rather than dykes) are favored because of neutral buoyancy. Heat is transferred from a hot sill either by conduction or by hydrothermal convection [Gulick, 1998; Harrison and Grimm, 2002] through the crust up to the bottom of the permafrost layer, as illustrated in Figure 1. The effect of a magmatic intrusion is modeled as the heterogeneous basal heating of the permafrost layer.

[14] The temperature and the size of the heating are set as follows. The extent is between $1 / 2$ and 2 times of the thickness of the permafrost layer, which is assumed to be on the order of kilometers. The central hot base is set as hundreds of Kelvin hotter than the surrounding baseline. 


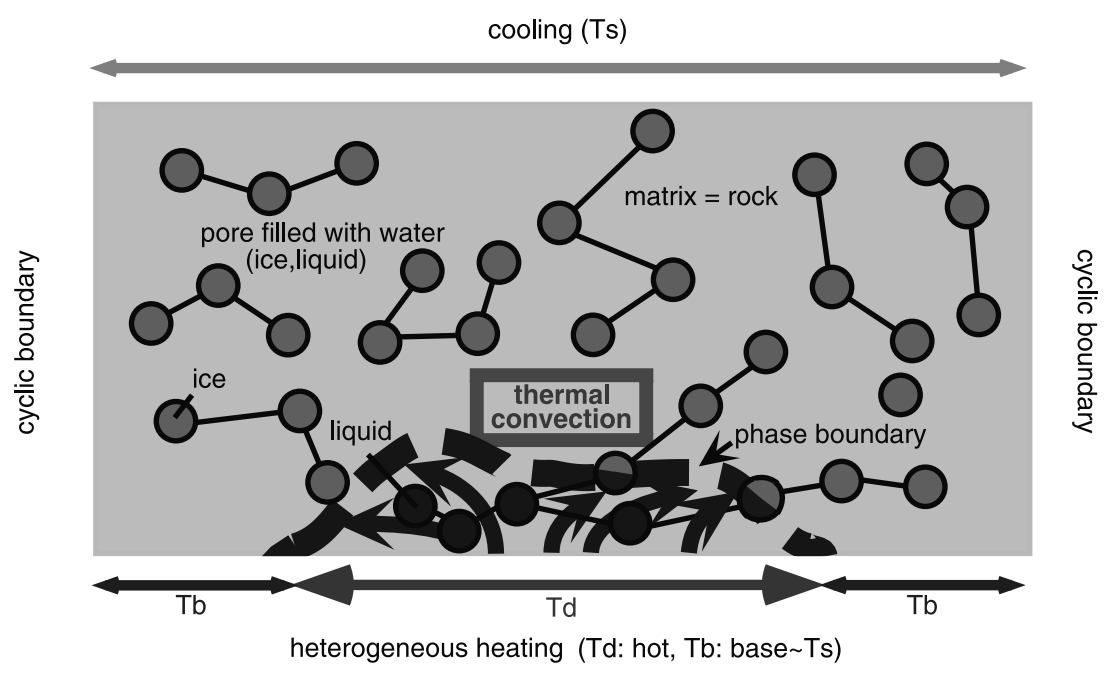

Figure 1. Modeling on the igneous melting of the permafrost layer. The permafrost can be treated as a porous medium in which the matrix is rock and the pores are filled with water ice. The effect of magmatic intrusion is expressed as the heterogeneous basal heating. When the temperature is above the melting point of water ice, the pore-ice melts. Thermal convection in the melted zone should then occur.

[15] The temperature of the bottom of the permafrost layer is considered fixed with time. Since the cooling time of the intrusive body takes considerably longer than the time required for melting ice, the temperature of the magma body is also considered constant. The temperature of the outer boundary of the intrusion experiences a large decrease in the short period just after emplacement. However, following this transient period, a quasi-steady-state condition is reached. We consider the situation after this transient cooling stage, which is the basis for our assumption that the temperature of the heated bottom region of the permafrost is constant.

[16] Initially, the transfer of heat through the permafrost occurs only by conduction. However, as the temperature increases, the permafrost eventually becomes warm enough for the ice within its pores to melt. At this point the porous medium consists of a mixture of two phases: the solid matrix with liquid in the pores. We assume a completely saturated situation and ignore the volume change associated with this phase change.

[17] The porosity of the Martian crust (volume percentage of ice phase in the permafrost) is not well known. Although the soils analyzed by Viking landers have a porosity of about 0.5 , this value only represents the state at the very top of the surface and must be a maximum value. Subsurface porosity would decrease with depth, although we use an averaged unique value for simplicity. In our calculation, considering the data of the permafrost on earth, we adopt a value ranging from 0.2 to 0.5 as the porosity.

\subsection{Mathematical Formulation}

\subsubsection{Thermal Convection in a Porous Medium}

[18] Melted region of the permafrost is made up of silicate rock and the pore space filled with water. The interconnection of the void space allows the fluid to migrate as a permeable flow. The flow quantities are in fact irregular on the pore scale, but they change in a regular manner on a macroscopic scale. We introduce the so-called Darcy velocity $\mathbf{u}$, the velocity of the fluid $\left(\mathbf{u}_{\mathrm{f}}\right)$ averaged over a representative volume element of the medium $\left(\mathbf{u}=\varepsilon \mathbf{u}_{\mathrm{f}}\right.$, $\varepsilon$ : porosity). We model the porous matrix/fluid mixture as incompressible and Boussinesq approximation is applicable.

[19] Basic equation sets are mass conservation, momentum conservation and energy conservation;

$$
\begin{gathered}
\nabla \cdot \mathbf{u}=0 \\
\nabla \mathrm{p}=-\mu / \mathrm{Ku}-\rho_{0} \mathbf{g} \beta\left(\mathrm{T}-\mathrm{T}_{0}\right) \\
(\rho \mathrm{c})_{\mathrm{m}} \partial \mathrm{T} / \partial \mathrm{t}+(\rho \mathrm{c})_{\mathrm{f}} \mathbf{u} \cdot \nabla \mathrm{T}=\nabla \cdot\left(\mathrm{k}_{\mathrm{m}} \nabla \mathrm{T}\right)
\end{gathered}
$$

where $\rho_{0}$ is the fluid density at a reference temperature $T_{0}, \beta$ is the coefficient of thermal expansion and $\mathbf{g}$ is the gravity. Here $\mu$ is the dynamic viscosity of the fluid, $p$ denotes an intrinsic pressure of the fluid, and t represents time. The coefficient $\mathrm{K}$ is called the specific permeability of the medium and is an invariant scalar in the case of an isotropic medium, which we suppose. In this application, $\mathrm{K}$ is given by the Carman-Kozeny equation:

$$
\mathrm{K}=\mathrm{D}_{\mathrm{p} 2}^{2} \varepsilon^{3} / 175(1-\varepsilon)^{2}
$$

where $D_{p 2}$ corresponds to an effective average particle diameter.

[20] In (3), the subscript $m$ refers to the porous medium, whose overall heat capacity per unit volume, $(\rho c)_{m}$, and overall thermal conductivity per unit volume, $\mathrm{k}_{\mathrm{m}}$, are given by

$$
\begin{gathered}
(\rho \mathrm{c})_{\mathrm{m}}=(1-\varepsilon)(\rho \mathrm{c})_{\mathrm{s}}+\varepsilon\left(\rho \mathrm{c}_{\mathrm{p}}\right)_{\mathrm{f}} \\
\mathrm{k}_{\mathrm{m}}=(1-\varepsilon) \mathrm{k}_{\mathrm{s}}+\varepsilon \mathrm{k}_{\mathrm{f}}
\end{gathered}
$$

where $\mathrm{c}$ is the thermal capacitance, the subscript $\mathrm{s}$ and $\mathrm{f}$ refer to the solid matrix and fluid pore, and $c_{p}$ is the specific heat capacity at constant pressure of the fluid. 


\subsubsection{Treatment of Phase Change: Enthalpy Method}

[21] The $\mathrm{H}_{2} 0$-filled pore space can change phase, while the matrix remains solid. The phase change from solid to liquid corresponds to the generation of liquid water in the permafrost.

[22] We adopt the enthalpy approach for simulating phase change process [Voller et al., 1990]. The method incorporates the latent heat into the enthalpy in the phase-changing area, so that we no longer need to explicitly treat the energy equations at the phase front. The advantage of this method over other numerical methods is an easy treatment of phase change with a complex moving boundary [Caldwell and Chan, 1998].

[23] In our simulation, latent heat effects are included once the temperature of a unit volume reaches the melting point. At this transition boundary, we assume a sufficiently small temperature interval to ensure the accuracy and stability of the solution. Over this narrow temperature range, the melt fraction changes from 0 to 1 in this phasechanging area, yielding a mixture of both the liquid and solid phases of water - a region we call the mushy zone.

[24] In case of a porous medium, the above enthalpy formulation is modified such that the region of phase change is restricted to the pore fluid. Following Beckermann and Viskanta [1988], we derive the energy equation in terms of the enthalpy method. A linear relationship of the melt fraction and the temperature is assumed. In the numerical simulation, the nondimensionalized equations have been solved by using the following parameters:

$$
\begin{gathered}
\nabla \cdot \mathbf{U}=0 \\
\nabla \mathbf{P}=-\kappa \mathbf{U}+\operatorname{Ra} \theta \mathbf{e}_{\eta}\left(\mathbf{e}_{\eta}: \text { a normal vertical vector }\right) \\
\Omega \partial \theta / \partial \tau+\mathbf{U} \cdot \nabla \theta=\nabla \cdot(\Lambda \nabla \theta)-1 / \text { Ste } \partial \delta / \partial \tau
\end{gathered}
$$

where

$$
\begin{aligned}
& \mathbf{U}=\mathrm{H} / \alpha_{1} \mathbf{u} ; \mathbf{P}=\mathrm{K} /\left(\rho_{1} \nu_{1} \alpha_{1}\right) \mathrm{p} ; \tau=\alpha / \mathrm{H}^{2} \mathrm{t} ; \\
& \theta=\left[\mathrm{T}-\left(\mathrm{T}_{\mathrm{d}}+\mathrm{T}_{\mathrm{s}}\right) / 2\right] /\left(\mathrm{T}_{\mathrm{d}}-\mathrm{T}_{\mathrm{s}}\right) ; \mathrm{X}=\mathrm{x} / \mathrm{H}, \mathrm{Y}=\mathrm{y} / \mathrm{H} \\
& \kappa=\mathrm{K}_{\varepsilon} / K(\delta) \\
& \Lambda=\mathrm{k}_{\mathrm{eff}} / \mathrm{k}_{\mathrm{l}} \\
& \Omega=\rho \mathrm{c} /\left(\rho_{l} c_{l}\right) \\
& \delta=\varepsilon \quad \text { for } \theta_{\mathrm{m}}+\Delta \theta \leq \theta \\
& \left.\varepsilon\left\{\left(\theta-\theta_{\mathrm{m}}+\Delta \theta\right) / 2 \Delta \theta\right)\right\} \quad \text { for } \theta_{\mathrm{m}}-\Delta \theta<\theta<\theta_{\mathrm{m}}+\Delta \theta \\
& 0 \quad \text { for } \theta \leq \theta_{\mathrm{m}}-\Delta \theta
\end{aligned}
$$

Here $\mathbf{U}, \mathbf{P}, \theta$, and $\tau$ are, respectively, dimensionless velocity, pressure, temperature and time. $\delta$ represents the fraction liquid in the volume element, $\mathrm{H}$ is the characteristic length, $\mathrm{x}$ is horizontal coordinate and $\mathrm{y}$ is vertical coordinate. $\mathrm{T}_{\mathrm{d}}$ and $T_{s}$ represent the bottom and the top temperature. The subscript 1 refers to the liquid water. Also described $\alpha$ is thermal diffusivity $\left(\alpha_{1}=\mathrm{k}_{1} /\left(\rho_{1} \mathrm{c}_{1}\right)\right)$ and $\nu$ is kinematic viscosity $\left(\nu_{1}=\mu_{1} / \rho_{1}\right)$ of the liquid. In (14), $\theta_{\mathrm{m}}$ is the dimensionless melting point and the value of $\Delta \theta$ is chosen to be sufficiently small to ensure stability.

[25] In this case, the following two nondimensional parameters are important to control the behavior of the system: the Rayleigh number (Ra) and the Stefan number (Ste) in (8) and (9).

$$
\begin{gathered}
\mathrm{Ra}=\mathrm{g} \beta_{\mathrm{l}} \mathrm{KH}\left(\mathrm{T}_{\mathrm{d}}-\mathrm{T}_{\mathrm{s}}\right) /\left(\nu_{1} \mathrm{\alpha}_{\mathrm{l}}\right) \\
\text { Ste }=\mathrm{c}_{\mathrm{l}}\left(\mathrm{T}_{\mathrm{d}}-\mathrm{T}_{\mathrm{s}}\right) / \Delta \mathrm{h}
\end{gathered}
$$

where $\Delta \mathrm{h}$ is the latent heat of fusion. The Rayleigh number represents the strength of thermally driven natural convection in a porous medium. The Stefan number represents the measure of thermal contribution of the phase change.

\subsubsection{Rayleigh Number}

[26] We have to conduct numerical simulations in a wide range of Ra because physical properties on Mars are quite uncertain and the suitable situations for simulating subsurface process on Mars are unknown. We start by assuming a minimum number of 40 to confirm the critical $\mathrm{Ra}$ for convection in a porous medium. Then considering the potential range of permafrost thickness, the possible values of crustal permeability, and probable temperature of the magma, we consider values of $\mathrm{Ra}$ up to $\sim 10^{3}$.

\subsubsection{Stefan Number}

[27] It is supposed that Ste ranges from order of $10^{-1}-$ $10^{1}$. We conduct calculations for two cases of Ste $=2$ and 5 .

\subsection{Assumed Initial and Boundary Conditions for Mars}

[28] Our numerical study is done for a rectangular grid whose aspect ratio (width/height) is 3 . Nodal points of $192 \times 64$ are used. The initial and boundary conditions for the dimensionless equations are as follows:

$$
\theta=\theta_{\mathrm{s}}, \mathbf{U}=0(\tau \leq 0)
$$

$$
\begin{aligned}
& \theta=\theta_{\mathrm{s}}(\mathrm{Y}=1,0 \leq \mathrm{X} \leq 1) \\
& \theta=\theta_{\mathrm{d}}(\mathrm{Y}=0,0.5-\mathrm{W} / 2 \leq \mathrm{X} \leq 0.5+\mathrm{W} / 2) \\
& \theta=\theta_{\mathrm{b}}(\mathrm{Y}=0, \mathrm{X} \leq 0.5-\mathrm{W} / 2,0.5+\mathrm{W} / 2 \leq \mathrm{X})
\end{aligned}
$$

$$
\mathbf{U} \cdot \mathbf{e}_{\eta}=0(\mathrm{Y}=0,1,0 \leq \mathrm{X} \leq 1)
$$

where $\mathrm{W}$ is the area of heterogeneous heating. Here $\theta_{\mathrm{s}}, \theta_{\mathrm{d}}$ and $\theta_{\mathrm{b}}$ are the dimensionless temperatures of, respectively, the top surface, central region of the bottom surface and the periphery of the bottom surface. The three principal variables are $\theta_{\mathrm{d}}, \theta_{\mathrm{b}}$ and $\mathrm{W}$. We simply set $\theta_{\mathrm{b}}$ as the same as that of the top surface $\theta_{\mathrm{s}}$ and can evaluate $\theta_{\mathrm{d}}$ that results from our assumed values of $\mathrm{Ra}$ and Ste. We also considered the case where $\theta_{\mathrm{b}}=0.5 \times\left(\theta_{\mathrm{s}}+\theta_{\mathrm{d}}\right)$, but the 


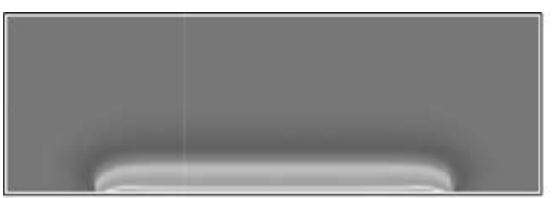

$\tau \sim 0.01$

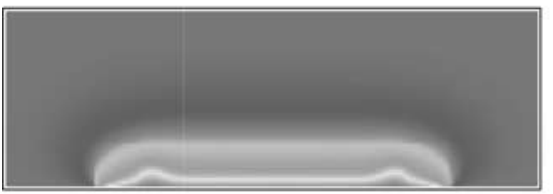

$\tau \sim 0.03$

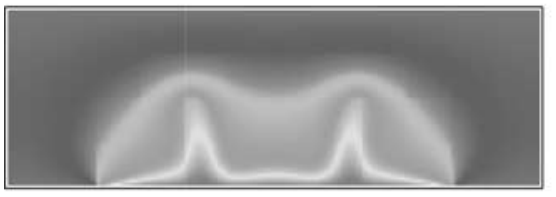

$\tau \sim 0.1$

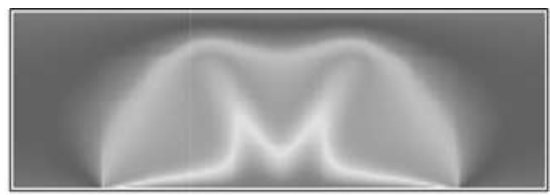

$\tau \sim 0.2$

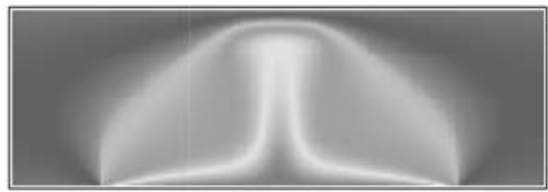

$\tau \sim 0.3$

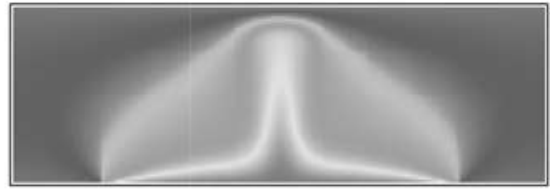

$\tau \sim 0.7$

Figure 2. Evolution of temperature field for the case; $\mathrm{Ra}=100$, Ste $=2, \varepsilon=0.2, \mathrm{~W}=2 \mathrm{H}$ and $\mathrm{A}=3$ (Ste: Stefan number, $\varepsilon$ : porosity, W: width of heterogeneous heating, H: thickness of the permafrost layer, A: aspect ratio). $\tau$ is dimensionless time, which is normalized by $\mathrm{H}^{2} / \alpha(\alpha$ : thermal diffusivity of water). At the initial stage $(\tau \sim 0.03)$, two rising hot zones appear at the edges of the heating area. At the later stage $(\tau \sim 0.1)$, two horn-shaped hot regions newly grow in the inner area. Then these two hot horns transform into a central single column $(\tau \sim 0.3)$. This hot column extends vertically and finally forms a stationary shape $(\tau \sim 0.7)$, remaining some distance below the surface.

results did not differ substantially from those calculated based on our first assumptions. So in the following, we discuss the effects of the Ra, Ste and $\mathrm{W}$ on the pattern of convection. For sidewalls, we utilize the cyclic boundary conditions for both velocity and temperature. The values of physical properties $(\rho$ : density, k: thermal conductivity, c: thermal capacitance) used in our calculation are the followings; $\rho_{\mathrm{H} 20}=1000\left[\mathrm{~kg} / \mathrm{m}^{3}\right], \rho_{\text {rock (soil) }}=2000\left[\mathrm{~kg} / \mathrm{m}^{3}\right]$, $\mathrm{k}_{\mathrm{water}}=0.561[\mathrm{~W} /(\mathrm{mK})], \mathrm{k}_{\mathrm{ice}}=2.2[\mathrm{~W} /(\mathrm{mK})], \mathrm{k}_{\text {rock (soil) }}=$ $0.14[\mathrm{~W} /(\mathrm{mK})], \mathrm{c}_{\text {water }}=4.21 \times 10^{3}[\mathrm{~J} /(\mathrm{K} \mathrm{kg})], \mathrm{c}_{\text {ice }}=1.962 \times$ $10^{3}[\mathrm{~J} /(\mathrm{K} \mathrm{kg})], \mathrm{c}_{\text {rock (soil) }}=0.8 \times 10^{3}[\mathrm{~J} /(\mathrm{K} \mathrm{kg})], \Delta \mathrm{h}=3.37 \times$ $10^{5}[\mathrm{~J} / \mathrm{kg}]$.

\section{Numerical Results on the Generation of Liquid Water}

\subsection{Shape of the Melted Zone}

[29] We investigate the extent of the melted region as a function of the Ra, size of the heating area and Ste. In the scheme of the enthalpy method, we can only see the melting boundary as a finite zone, which we call the "mushy zone." Hereafter we refer to the melt zone as the region where the average temperature within a volume element is above the melting point. This corresponds to the region where the melt fraction is above 0.5 (see section 2). Thus, defined the melt zone covers almost half of the mushy zone plus the entire liquid zone.

\subsubsection{Rayleigh Number Dependency}

[30] We first exhibit the results of the simulation performed with a following set of parameters; $\mathrm{Ra}=100$,
Ste $=2, \varepsilon=0.2, \mathrm{~W}=2 \mathrm{H}$, in Figure 2 . The melting process is characterized by the following steps, where $\tau$ represents dimensionless time.

[31] At the initial stage $(\tau \sim 0.03)$, two rising hot zones appear at the edges of the heating area. At the later stage $(\tau \sim 0.1)$, these zones disappear and two horn-shaped hot regions newly grow in the inner area. Then these two hot horns transform into a central single column $(\tau \sim 0.3)$. This hot column extends vertically and finally forms stationary shape $(\tau \sim 0.7)$ remaining some distance to the top surface.

[32] With the increase of Ra, the pattern exhibits a quite different appearance both at the transient stage and at the final stage. Figure 3 shows the results at $\mathrm{Ra}=700$. Other parameters are not changed. This parameter set is used as a basic reference state and, based on this, we conduct parameter studies hereafter. At the initial period, a number of hot narrow columns exist $(\tau \sim 0.01)$. The morphology is similar to that of Benard convection. As time advances, the multiple columns disappear to form a single central column $(\tau \sim 0.15)$ as in the case for $\mathrm{Ra}=100$. The hot zone extends vertically with time and reaches very near to the top surface. Finally, the hot zone spreads laterally $(\tau>0.17)$.

[33] The other results of simulation performed at various $\mathrm{Ra} ; \mathrm{Ra}=150,400,1000,1200$ are shown in Figure 4 with description of each applied conditions and parameters.

[34] The results of these simulations differ in two ways: the number of columns at the initial stage and the shape of melted zone in the steady state. We see, at the larger Ra, a larger number of hot columns are generated during the 


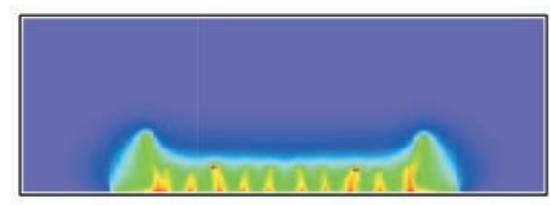

$\tau \sim 0.01$

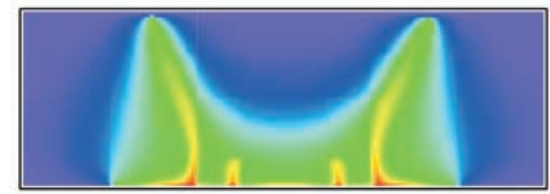

$\tau \sim 0.03$

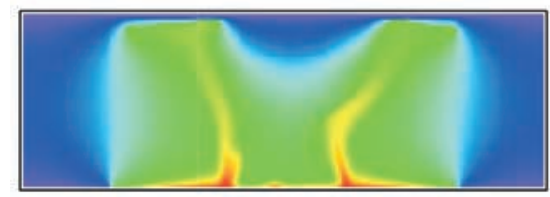

$\tau \sim 0.1$

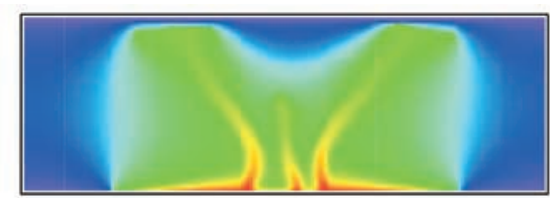

$\tau \sim 0.13$

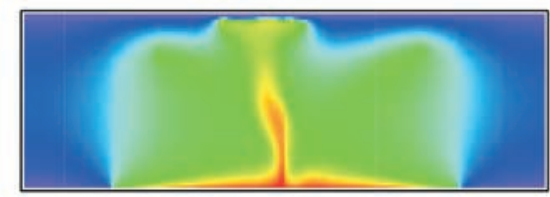

$\tau \sim 0.15$

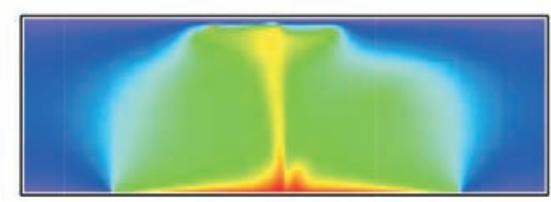

$\tau \sim 0.17$

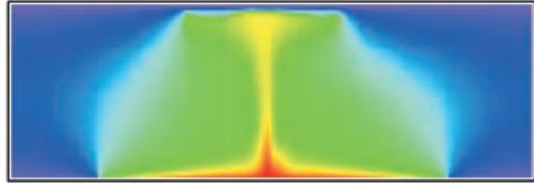

$\tau \sim 0.2$

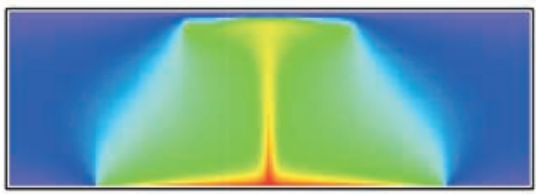

$\tau \sim 0.7$

Figure 3. Evolution of temperature field at $\mathrm{Ra}=700$. Other parameters are the same as those in Figure 2. This parameter set is used as a basic reference state. $\tau$ represents nondimensional time. At the initial period $(\tau \sim 0.01)$, a number of hot narrow columns exist. As time advances, the multiple columns disappear to form a single central column $(\tau \sim 0.15)$ as in Figure 2 at $\mathrm{Ra}=100$. The hot zone extends vertically with time and extends almost to the surface. Lastly, the hot zone spreads laterally $(\tau>0.7)$ in equilibrium state. The zone which is about a size larger than the yellow region corresponds to the melted zone.

initial period and, in the steady state, the hot zone extends more laterally around its top region, like a plume with vertical column. As a reference, we also show the numerical result in the case of conduction only (Figure 4e). For comparison, all the other parameters, other than the Ra, are held constant (Ste $=2, \varepsilon=0.2, \mathrm{~W}=2 \mathrm{H}$ ). The temperature field for the conductive case is quite different from that for the convective case; the hot region $(=$ the melted zone) stays at the bottom and extends mainly only laterally. No substantial vertical column is observed. This is the same shape that McKenzie and Nimmo [1999] describe.

[35] The results we have described so far are about the characteristics of the temperature field. The discussion is valid for the melting state, because the zone directly reflects the temperature fields in the enthalpy method.

[36] We conclude that thermal convection in the melted zone substantially alters the morphology of the melting region and its growth timescale. Thermal convection in the melt enhances and localizes vertical heat transfer, which is almost impossible by conduction. We call the characteristic of this heat transport "focusing."

[37] The strength of the focusing is displayed as the shape of the melted zone and it sensitively depends on the Ra. At large $\mathrm{Ra}(\mathrm{Ra}>400)$, the strong focusing of heat transfer seems to shape the melted zone into a plume-like structure, which is composed of a vertical column and laterally extending umbrella at the top. In the case of small Ra $(\mathrm{Ra}<100)$, only a vertical column exists with no umbrella region.

[38] This indicates that, at large $\mathrm{Ra}$, a high concentration of liquid water near the top surface is expected. Under Martian conditions, the value of Ra may range from several hundred to greater, considering the combination of uncertainties of $\mathrm{K}, \mathrm{H}$ and $\left(\mathrm{T}_{\mathrm{d}}-\mathrm{T}_{\mathrm{s}}\right)$. The above plume-like feature is very important in formation of fluvial features on the surface, which we discuss in section 4.

\subsubsection{Heating Area Dependency}

[39] Variations in the heating area also control the morphology of the melt region. Up to now, the bottom heating area at the center is assumed as $\mathrm{W}=2 \mathrm{H}(\mathrm{H}$ is the thickness of a porous medium). However, we also simulated the cases of $\mathrm{W}=1 / 2 \mathrm{H}$ and $\mathrm{H}$. As expected, the larger the heating area is, the more heat can be transferred, which results in expansion of the hot zone. But the shape of the melted region in the steady state is different according to the heating area. For larger heating areas, the melt zone extends more laterally at its top, like a plume with vertical column.

\subsubsection{Stefan Number Dependency}

[40] Figure 5 shows the case for Ste $=5$, which is 2.5 times as large as the basic case. Other parameters are not changed. A well-defined umbrella region is no longer observed; instead, the melt region extends over quite a large area of the permafrost layer.

\subsection{Production of Meltwater}

\subsubsection{Rayleigh Number Dependency}

[41] The production of meltwater as a function of time and $\mathrm{Ra}$ is illustrated in Figure 6.

[42] The conduction case, which is illustrated as a reference, shows only a gradual rise in melt volume with time. This trend is essentially the same as that found by McKenzie and Nimmo [1999].

[43] At $\mathrm{Ra}=100$, when the convective heat transfer is considered, the production of water shows quite a different 


$$
\tau \sim 0.01
$$
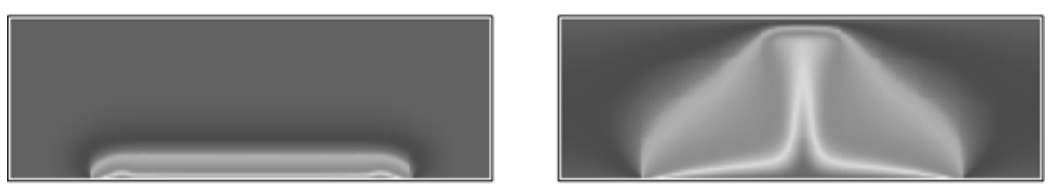

(a) $\mathrm{Ra}=150$
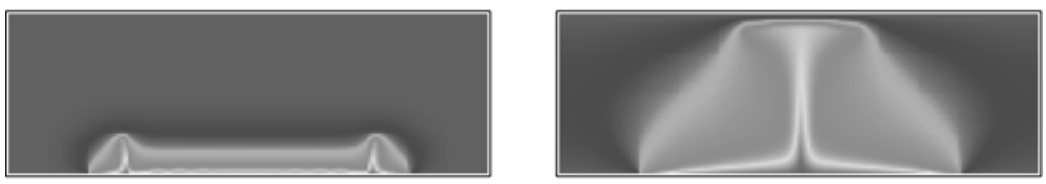

(b) $\mathrm{Ra}=400$
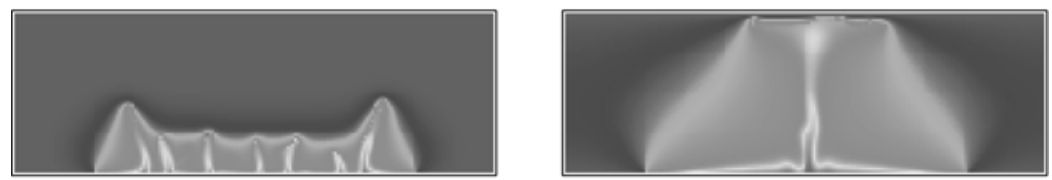

(c) $\mathrm{Ra}=1000$
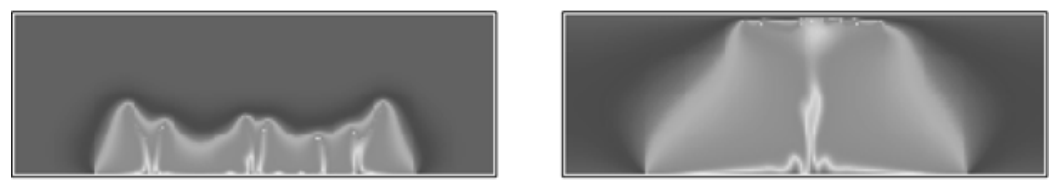

(d) $\mathrm{Ra}=1200$
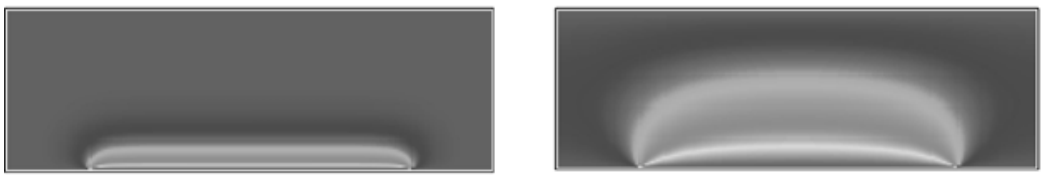

(e) conduction case

Figure 4. Evolution of temperature field at various $\mathrm{Ra}$; $\mathrm{Ra}=$ (a) 150, (b) 400, (c) 1000, (d) 1200. Other than the $\mathrm{Ra}$, parameters are the same as those for the basic reference state in Figure 3. $\tau$ represents nondimensional time. Left sides are the snapshots at initial stage $(\tau \sim 0.01)$ and right sides are those in steady state $(\tau \sim 0.7)$. For comparison, temperature fields for the conduction case (e) are also shown. Note particularly significant difference in the morphology of the melted zone in the steady state.

trend. At the initial or middle stage, the melt volume increases abruptly with time. This stage corresponds to the state where the central stationary column has not yet formed. The volume is almost twice or more times as large as that for the conduction case at the corresponding time. After reaching the maximum, the value of melt volume decreases and settles down to a stationary value, where the melting system seems to reach an equilibrium state. Compared with the case of conduction, efficient heat transfer results in a considerable increase of water volume especially for the case of $\mathrm{Ra}=100$.

[44] As the $\mathrm{Ra}$ increases, it takes less time to reach the peak melt volume and achieve a steady state. This indicates that more active convection works to enhance heat transfer toward the stable state in a shorter time.
[45] We note that the final water volume available is smaller at the larger Ra. This must be closely related to the shape of melt zone. As we see in the previous figures, at larger $\mathrm{Ra}$, narrower columns are formed (than those at smaller Ra) in steady state, although they extend more vertically. Significant heat transfer is therefore limited to the narrow column.

\subsubsection{Heating Area Dependency}

[46] Figure 7 illustrates development of meltwater volume for several cases of heating area: $\mathrm{W}=1 / 2,1,2 \mathrm{H}$. As one would intuitively expect, the greater the extent of the heating area, the greater the volume of water produced.

\subsubsection{Stefan Number Dependency}

[47] Figure 8 illustrates the production of meltwater for Ste $=5$. A drastic increase compared with the basic case is 


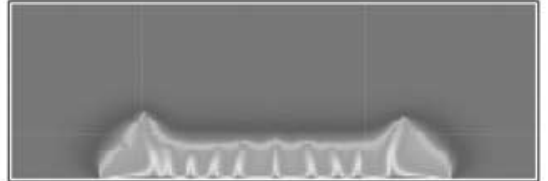

$\tau \sim 0.02$

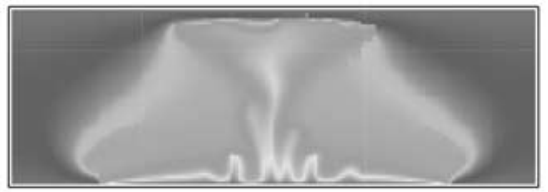

$\tau \sim 0.1$

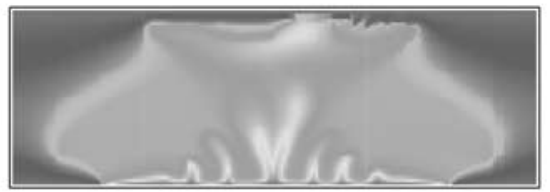

$\tau \sim 0.2$

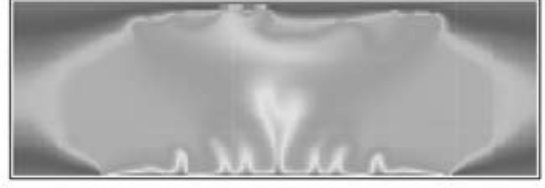

$\tau \sim 0.3$

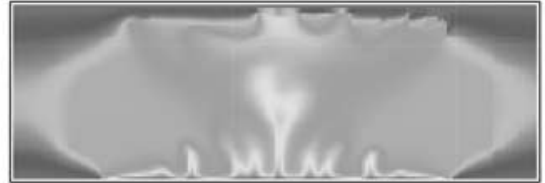

$\tau \sim 0.7$

Figure 5. Evolution of temperature field at $\mathrm{Ste}=5$. Other parameters are the same as those for the basic reference state in Figure 3.

observed, which results from the larger difference of the characteristic temperatures in the system.

\subsubsection{Water Flux}

[48] From a review of the results summarized in Figures $6-8$, we see that those changes in parameters to promote more efficient heat transfer (such as larger values of Ra, heating area, and Ste) clearly result in an increase in water generation vs. the conduction reference-case. The dimensional values of these variables critically depend on the characteristic length scale of the thickness of the permafrost

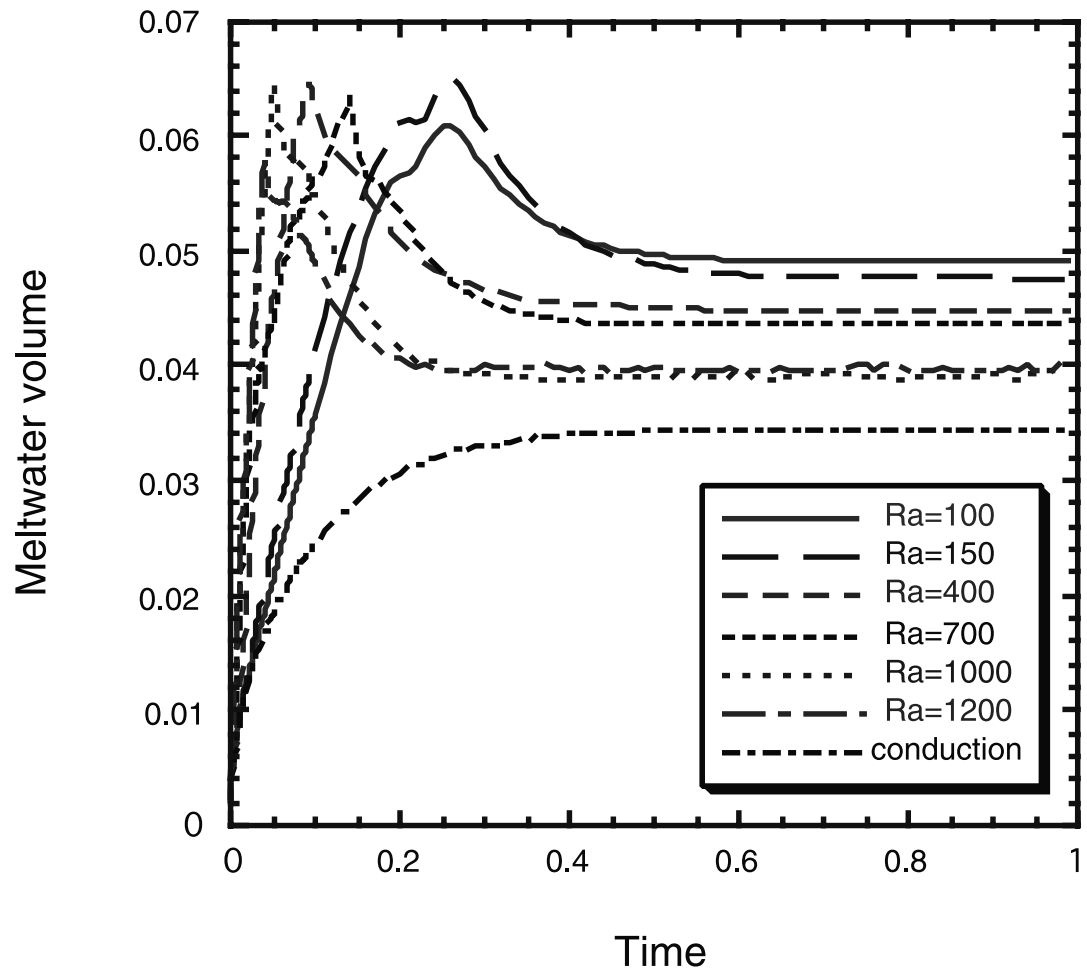

Figure 6. Production of meltwater as a function of time for various Ra: 100-1200. The pure conduction case is illustrated as a reference, showing only a gradual rise in water volume with time. This contrasts with an abrupt rise in meltwater production associated with the initial and middle stages of convection. As the $\mathrm{Ra}$ increases, it takes less time to reach both the peak point of the melt volume production and ultimate steady state. Volume is normalized by $3 \mathrm{H}^{2} \mathrm{D}(\mathrm{H}$ : thickness of the permafrost layer, D: the depth). Time is nondimensionalized by $\mathrm{H}^{2} / \alpha(\alpha$ : thermal diffusivity of water). 


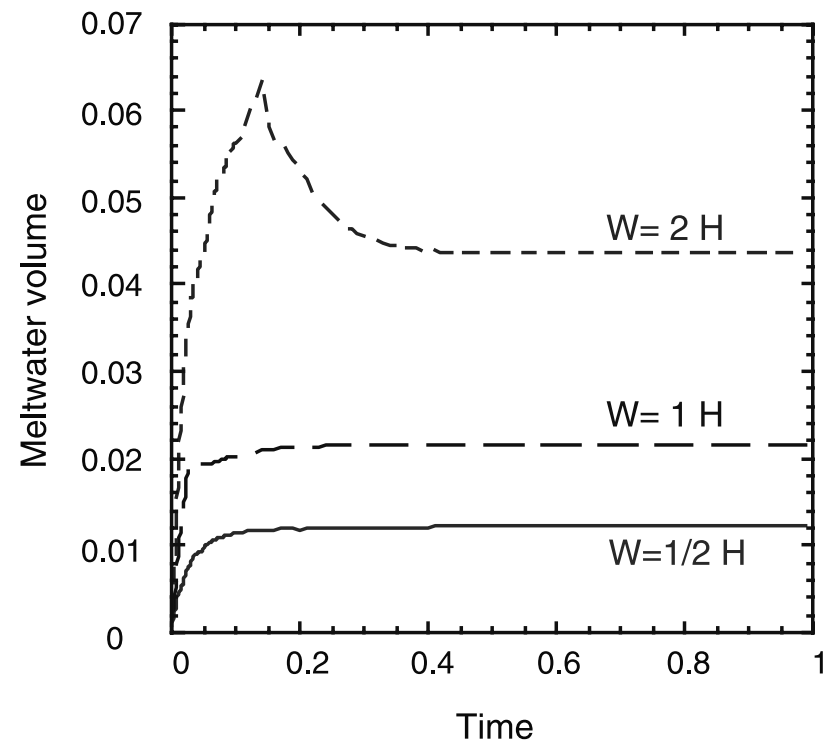

Figure 7. Development of meltwater with time for changing extent of heating area: $\mathrm{W}=1 / 2 \mathrm{H}, \mathrm{H}, 2 \mathrm{H}$. With a larger heating area, more water is produced. Volume is normalized by $3 \mathrm{H}^{2} \mathrm{D}(\mathrm{H}$ : thickness of the permafrost layer, D: the depth). Time is nondimensionalized by $\mathrm{H}^{2} / \alpha(\alpha$ : thermal diffusivity of water).

layer, H, and its horizontal depth, D. Assuming $\mathrm{H}$ and D as several kilometers, the max rate would be $10^{-3} \mathrm{~m}^{3} / \mathrm{s}$ for various cases considered here.

\section{Implication for Surface Features}

[49] Based on our calculations of subsurface melting, we now explore possible surface manifestations of the process. We first consider the potential transport of subsurface meltwater up to the surface. Next, we discuss the expected formation of surface features resulting from the discharge of this water. Several observed characteristics of chaotic terrain appear consistent with our results.

\subsection{Expected Surface Features: A Consistent Scenario}

\subsubsection{Compaction and Segregation of the} Thawed Permafrost

[50] The melted zone of the permafrost consists of a rock matrix and interconnected water-filled pores. In such a mixture of two phases, the compaction of the solid phase (or segregation of the liquid phase) seems likely to occur in due time if sufficient lithostatic pressure is developed under gravity. We notice here again that the shape of the zone resembles a plume structure consisting of a thick column of meltwater and thawed permafrost. Within this region, we believe that compaction would cause the meltwater to segregate in the laterally extended upper region just below the surface.

[51] In evaluating this process, three kinds of timescales are important; the compaction time $\left(\mathrm{t}_{\mathrm{C}}\right)$, the melting time of the permafrost layer $\left(t_{\text {melting }}\right)$ and the cooling time of the magma $\left(t_{\text {cooling }}\right)$. The relationship between these three timescales exerts a strong influence on the extent to which compaction will occur or not. To calculate the compaction time, we follow McKenzie [1984] and Sumita et al. [1996].
[52] The compaction time, the time taken for the Darcy flow (velocity $\mathrm{V}_{\mathrm{D}}$ ) to flow the distance of the compaction length $\left(\mathrm{L}_{\mathrm{C}}\right)$, is obtained as follows:

$$
\begin{gathered}
\mathrm{t}_{\mathrm{C}}=\mathrm{L}_{\mathrm{C}} / \mathrm{V}_{\mathrm{D}}=\left[\left(\mathrm{K}_{\phi 0} \psi_{0}\left(\eta_{\phi 0} * / \eta_{\mathrm{f}}\right)\right]^{1 / 2} /\left[\left(\mathrm{K}_{\phi 0} / \eta_{\mathrm{f}}\right) \psi_{0} \Delta \rho g\right]\right. \\
=1 / \Delta \rho g\left(\eta_{\phi 0} * \eta_{\mathrm{f}} / \mathrm{K}_{\eta 0} \psi_{0}\right)^{1 / 2}
\end{gathered}
$$

where $\mathrm{K}_{\phi}$ is the permeability, $\eta_{\mathrm{f}}$ is the shear viscosity of melt, $\psi$ is solidity ( $=1-\varepsilon, \varepsilon$ : porosity), $\Delta \rho$ is density difference between melt and solid and $g$ is gravity. Here the subscript 0 denotes the value at the surface. $\eta_{\phi}{ }^{*}$ is the effective bulk viscosity of the matrix, which can be derived as [Sumita et al., 1996]

$$
\eta_{\phi}^{*}=4(1-\varepsilon) / 3 \varepsilon \eta_{\mathrm{s}}
$$

Here $\eta_{\mathrm{s}}$ is the shear viscosity of the solid.

[53] Assuming $\Delta \rho \sim 2000\left[\mathrm{~kg} / \mathrm{m}^{3}\right], \mathrm{g} \sim 3\left[\mathrm{~m}^{2} / \mathrm{s}\right], \sim 10^{-14}$ $\left[\mathrm{m}^{2}\right]<\mathrm{K}_{\phi 0}<\sim 10^{-11}\left[\mathrm{~m}^{2}\right], 0.2<\varepsilon<0.5$ and putting $\eta_{\mathrm{s}}$ as the value for sediment of $10^{21}$ [Pa s] [Sumita et al., 1996], the compaction time $t_{\mathrm{C}}$ is estimated as $\sim 10^{11}-10^{13}$ seconds $\left(\sim 10^{-2}-10^{0} \mathrm{Myr}\right)$.

[54] In this estimation, the most uncertainty comes from the bulk viscosity calculated from the formula of Sumita et al. [1996], a formula that appears theoretically sound but for which there has been no experimental verification. Furthermore, the referred value of $\eta_{\mathrm{s}}$ may only be valid for the sediment and is probably overestimated for the matrix of the permafrost in our situation.

[55] The cooling time of the magmatic body is roughly given by the conduction timescale. Assuming a scale of $\sim 10^{1-2} \mathrm{~km}$, the time required for cooling would be

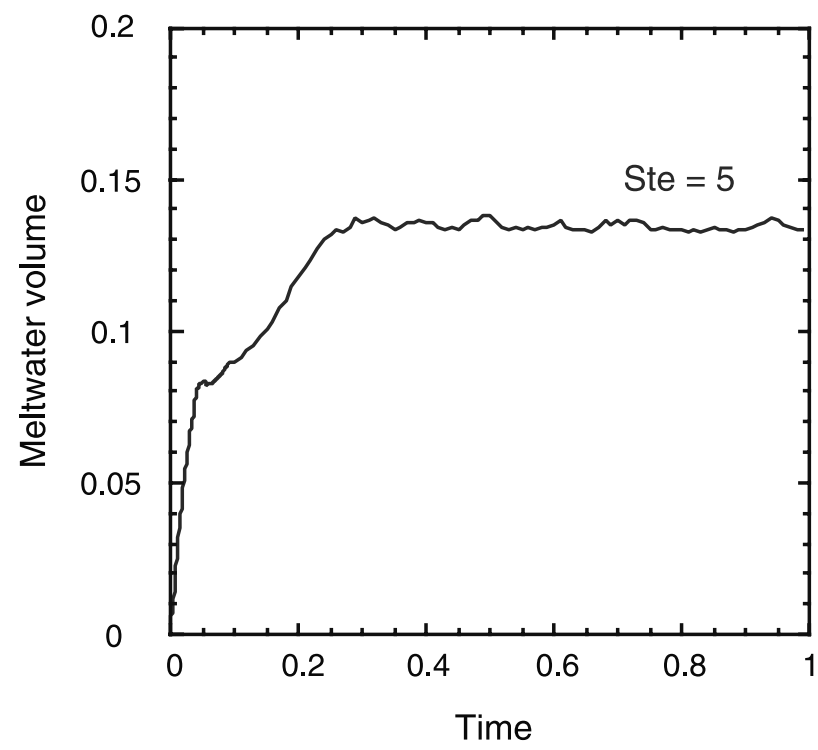

Figure 8. Development of meltwater volume with time at $\mathrm{Ste}=5$. Volume is normalized by $3 \mathrm{H}^{2} \mathrm{D}(\mathrm{H}$ : thickness of the permafrost layer, D: the depth). Time is nondimensionalized by $\mathrm{H}^{2} / \alpha$ ( $\alpha$ : thermal diffusivity of water). 


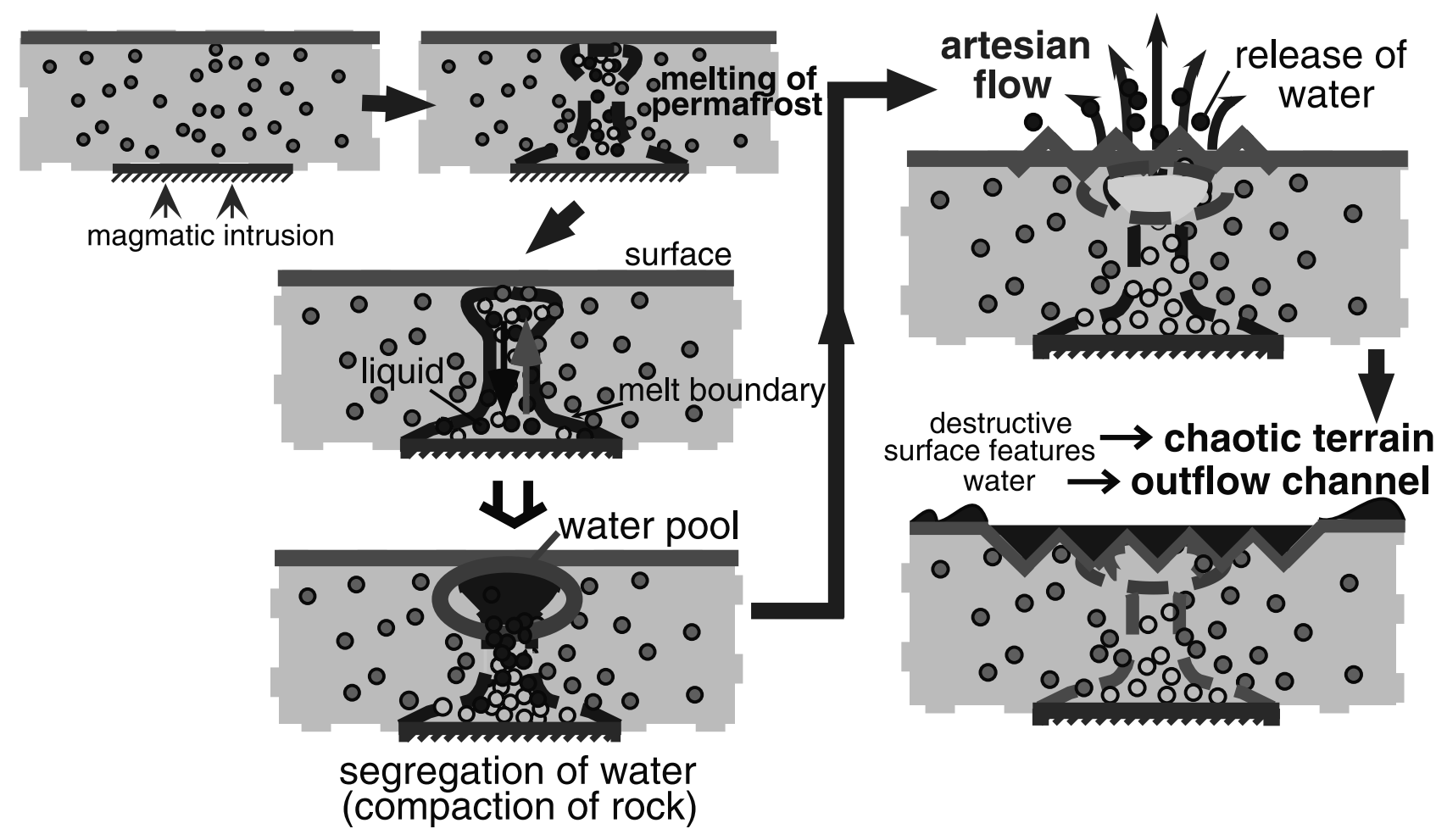

Figure 9. A scenario for the origin of chaotic terrain and outflow channels. A magmatic intrusion heats the permafrost layer from the bottom. The resultant melted zone resembles a mushroom-shaped plume. Compaction of the rock matrix is expected to result in the migration and pooling of water at the top of the melted zone (see the left bottom). The lithospheric pressure exerted by the overlying rock would create hydrostatic pressures sufficient to cause an artesian discharge to the surface. Such an eruptive flow event would catastrophically release subsurface water up to the surface, causing considerable collapse of the overlying surface and resulting in the extensive fluvial erosion evident in the morphology of the outflow channels.

$\sim 10$ Myr. In section 3, the nondimensional melting time was found to be 0.7 or less, which should correspond to the dimensional time of the order of $\sim 1$ Myr or less.

[56] With these parameters, we have the following relation in timescale:

$$
\mathrm{t}_{\text {melting }} \sim \mathrm{t}_{\mathrm{C}(\text { compaction })}<\mathrm{t}_{\text {cooling }}
$$

[57] This means the compaction would occur on a comparable timescale to the progress of the melting, which is clearly before the cooling of the magma. The relationship between the timescales of compaction and melting are difficult to determine quantitatively because of the large uncertainties associated with the various parameters. However, we see that $\mathrm{L}_{\mathrm{C}}$ must be of the order of kilometers. This indicates that compaction should not occur until the vertical length of the melt zone reaches near the top of the permafrost layer and thereafter the process would progress in a comparatively short timescale. Therefore, we consider that the rock matrix would not compact quickly before the progress of melting and would not disturb the convective heat transport. The expected result is that the upward segregation of water would proceed during and after the melting of the poreice to make a water pool very near the surface, as illustrated at the left bottom of Figure 9.

\subsubsection{Artesian Flow of Water to the Surface}

[58] Assuming a water pool next to the surface, the lithospheric pressure of the surrounding rock would exceed the hydrostatic pressure of the water and result in artesian flow. It is expected that such an eruptive flow event would catastrophically release subsurface water in a short time, accompanied by considerable collapse of the surface, thus explaining both the formation of the outflow channels and the creation of chaotic terrain.

[59] The extent of the collapsed surface, corresponding to the scale of the discharge event, must reflect the original subsurface situation of the water pooling, which in turn directly corresponds to the melting condition of the permafrost layer. What control the melting process are the Rayleigh number, the Stefan number and heating area. The scale of the collapse for probable set of parameters; $\mathrm{Ra}=700$, porosity $=0.2$, Ste $=2$ and $\mathrm{W}=2 \mathrm{H}$ is estimated as the same order as the thickness of the permafrost layer. Assuming the thickness of the permafrost layer as $10^{0}-10^{1}$ $\mathrm{km}$, the subsurface water extends laterally on the same scale. The expected size of the surface collapse might be somewhat enlarged by explosive release of the water. So in this basic parameter set, the extent of surface disruption will be $\geq 10^{\circ} \mathrm{km}$. A larger Rayleigh number or wider heating area should result in a more extended surface collapse in lateral scale due to a larger subsurface pool. 
[60] We summarize the probable formation of surface features in Figure 9.

\subsection{Geomorphology of the Observed Features}

[61] Outflow channels, chasma and chaos are located close to each other where volcano/ice interaction is quite probable as described in section 1. Here we compare these geomorphological features with the scenario presented above.

\subsubsection{Chaotic Terrains}

[62] Chaos or chaotic terrain (Figure 10) is where the ground has seemingly collapsed to form broad areas of jostled blocks and knobs that may lie as much as $1-2 \mathrm{~km}$ below the surrounding terrain [Carr, 1996]. The location of most chaotic terrains is between the canyon system Valles Marineris and the Chryse basin, extending from $20^{\circ} \mathrm{S}$ to $5^{\circ} \mathrm{N}$, and $15^{\circ} \mathrm{W}$ to $50^{\circ} \mathrm{W}$, being entirely surrounded by heavily cratered terrains. The individual chaos is on a lateral scale of $\sim 10^{2} \mathrm{~km}$, although its distinction from the outflow channel itself is often ill defined.

[63] The nature of the chaotic terrain clearly indicates that the surface collapse has occurred there [Carr, 1979, 1996; Lucchitta et al., 1994] and almost all occurrences are located in the headwater regions of the outflow channels. Although the observed scale of some chaotic terrain seems somewhat larger than the scale expected from a single artesian flow event, there is evidence that suggests that these may have been formed by the coalescence of chaos from multiple events.

\subsubsection{Outflow Channels}

[64] Outflow channels are typically $\sim 10-10^{2} \mathrm{~km}$ across and $\sim 10^{2}-10^{3} \mathrm{~km}$ in length [Baker et al., 1992].

[65] From the dimensions of the channels, their flow rates are estimated to be quite large, in the range of $10^{7}-10^{9} \mathrm{~m}^{3} / \mathrm{s}$ [Baker, 1982; Komar, 1979; Robinson and Tanaka, 1990]. Because the production rate of meltwater in our simulations is far less than this amount, this suggests that the water produced by the igneous melting of ground ice is not enough to form the channels unless it was first pooled in large reservoirs and suddenly released. McCauley [1978] was the first to propose this idea, but he assumed the source was a surface lake. However, as demonstrated in section 4.1, the geothermal melting and subsurface pooling of water, induced by compaction and segregation within the melted zone, appear to offer a more viable possibility, where the subsequent release of the pooled water by artesian flow would then have enabled the flood event. Our results show that the total mass of water could reach $>10^{9-10} \mathrm{~m}^{3}$ in the case where the thickness of the permafrost layer is $2 \mathrm{~km}$, yielding a potential discharge sufficient to match the $\sim 10^{7-9} \mathrm{~m}^{3} / \mathrm{s}$ inferred from the morphometric characteristics of the outflow channels. Although more speculative, the geothermal melting of icerich permafrost might also explain the origin of some of the chasmata and pit-chains associated with Valles Marineris [Lucchitta et al., 1992; Spencer and Fanale, 1990].

\section{Summary}

[66] The igneous melting of the permafrost layer is largely controlled by the size and vigor of thermal convection in the melted zone. We have quantitatively assessed the effect of convection on enhancing and focusing heat transfer, finding that the resulting melt zone extends up to just beneath the surface, creating a plume with a mushroom- (a)

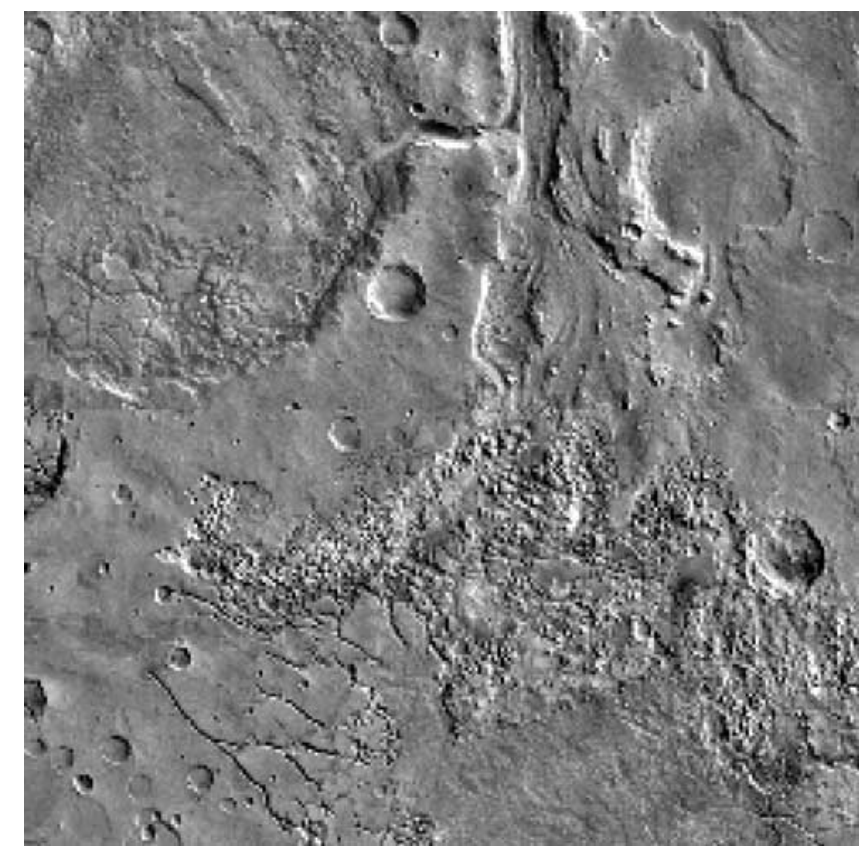

(b)

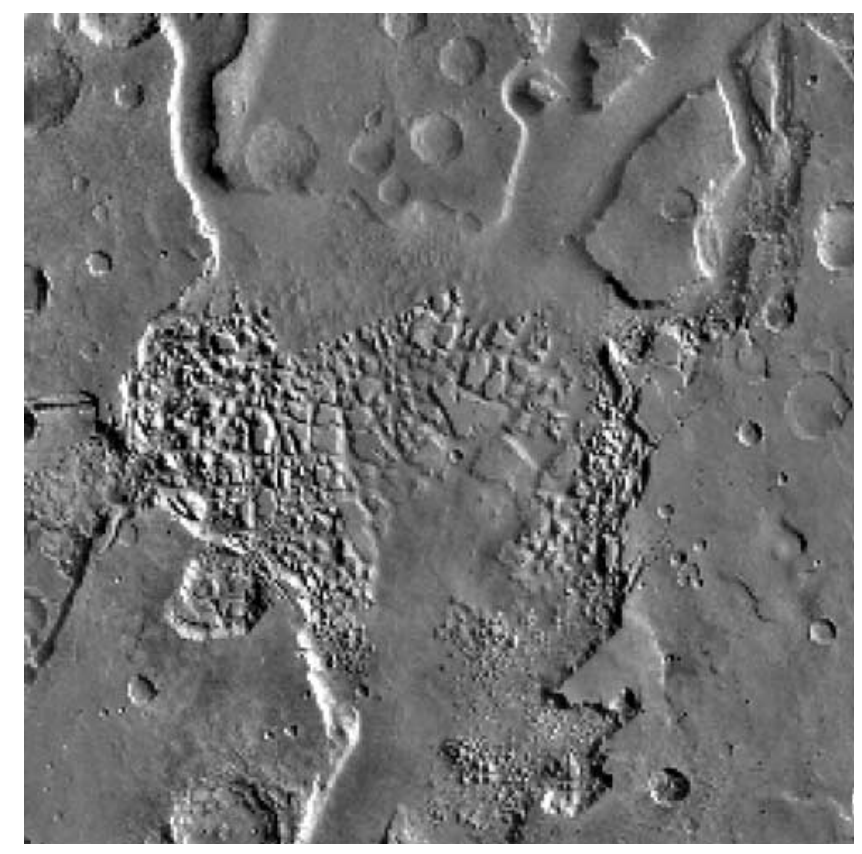

Figure 10. Examples of chaotic terrains in (a) Iani Chaos $\left(2^{\circ} \mathrm{S}, 18^{\circ} \mathrm{W}\right)$ and (b) Hydraotes Chaos $\left(1^{\circ} \mathrm{N}, 34^{\circ} \mathrm{W}\right)$ from Viking Orbiter image mosaic (sinusoidal projection). Image courtesy of NASA Planetary Data System Imaging Node, U. S. Geological Survey, Flagstaff, AZ.

like structure. The resulting volume of meltwater is several times as much as that expected in the conduction case. These two characteristics mean that substantial amounts of water could exist very near the surface. Subsequent compaction should result in the expulsion of the water from the rock matrix to form a pool at the top of the melt zone. The artesian discharge of this water, accompanied by the col- 
lapse and disruption of the overlying surface, might then explain the outflow channels and chaotic terrain. Such a scenario appears to explain much of the observational evidence.

\subsection{Observational Tests of Our Model in Future Missions}

[67] The validity of this scenario can be tested by a search for hydrothermal minerals around chaotic terrain and chasma. Hydrothermal minerals typically have spectral features in the near-infrared. Pyroxene and sulfates, which are likely to be contained in any Martian magma, have their absorption bands in the same range. Therefore, evidence of magma/ice interactions should be detectable by a nearinfrared spectrometer with sufficient resolution.

[68] Acknowledgments. We thank Horton Newsom and an anonymous referee for thoughtful comments and discussion. We also thank Stephen M. Clifford, whose suggestions improved the clarity of the descriptions very much.

\section{References}

Baker, V. R., The Channels of Mars, Univ. of Tex. Press, Austin, 1982.

Baker, V. R., and D. J. Milton, Erosion by catastrophic floods on Mars and Earth, Icarus, 23, 27-41, 1974.

Baker, V. R., M. H. Carr, V. C. Gulick, C. R. Williams, and M. S. Marley, Channels and Valley networks, in Mars, edited by H. H. Kieffer et al., pp. 493-522, Univ. of Ariz. Press, Tuscon, 1992.

Barlow, N. G., Crater size-frequency distribution and a revised Martian chronology, Icarus, 75, 285-305, 1988.

Beckermann, C., and R. Viskanta, Natural convection solid/liquid phase change in porous media, Int. J. Heat Mass Transfer, 31, 35-46, 1988.

Caldwell, J., and C.-C. Chan, Numerical solutions of the Stefan problem by the enthalpy method and the heat balance integral method, Num. Heat Transfer, 33, 99-117, 1998.

Carr, M. H., Formation of Martian flood features by release of water from confined aquifers, J. Geophys. Res., 84, 2995-3007, 1979.

Carr, M. H., Water on Mars, Oxford Univ. Press, New York, 1996.

Clifford, S. M., A model for the hydrologic and climatic behavior of water on Mars, J. Geophys. Res., 98, 10,973-11,016, 1993.

Gulick, V. C., Magmatic intrusions and a hydrothermal origin for fluvial valleys on Mars, J. Geophys. Res., 103, 19,365-19,387, 1998.
Harrison, K. P., and R. E. Grimm, Controls on Martian hydrothermal systems: Application to valley network and magnetic anomaly formation, J. Geophys. Res., 107(E5), 5025, doi:10.1029/2001JE001616, 2002.

Komar, P. D., Comparisons of the hydraulics of water flows in Martian outflow channels with flows of similar scale on Earth, Icarus, 37, 156$181,1979$.

Lucchitta, B. K., A. S. McEwen, G. D. Clow, P. E. Geissler, R. B. Singer, R. A. Shultz, and S. W. Squyres, The canyon system on Mars, in Mars, edited by H. H. Kieffer et al., pp. 453-492, Univ. of Ariz. Press, Tuscon, 1992.

Lucchitta, B. K., N. K. Isbell, and A. Howington-Kraus, Topography of Valles Marineris: Implications for erosional and structural history, J. Geophys. Res., 99, 3783-3798, 1994.

Max, M. D., and S. M. Clifford, Initiation of Martian outflow channels: Related to the dissociation of gas hydrate?, Geophys. Res. Lett., 28, 1787-1790, 2001.

McCauley, J. F., Geologic map of the Coprates Quadrangle of Mars, U.S. Geol. Surv. Misc. Invest. Ser., MapI-897, 1978.

McKenzie, D. P., The generation and compaction of partially molten rocks, J. Petrol., 25, 713-765, 1984.

McKenzie, D., and F. Nimmo, The generation of Martian floods by the melting of ground ice above dykes, Nature, 397, 231-233, 1999.

Robinson, M. S., and K. L. Tanaka, Magnitude of a catastrophic flood event at Kasei Valles, Mars, Geology, 18, 902-905, 1990.

Rossbacher, L. A., and S. Judson, Ground ice on Mars: Inventory, distribution, and resulting landforms, Icarus, 45, 39-59, 1981.

Spencer, J. R., and F. P. Fanale, New models for the origin of Valles Marineris closed depressions, J. Geophys. Res., 95, 14,301-14,313, 1990.

Squyres, S. W., The distribution of lobate debris aprons and similar flows on Mars, J. Geophys. Res., 84, 8087-8096, 1979.

Squyres, S. W., and M. H. Carr, Geomorphic evidence for the distribution of ground ice on Mars, Science, 231, 249-252, 1986.

Sumita, I., S. Yoshida, M. Kumazawa, and Y. Hamano, A model for sedimentary compaction of a viscous medium and its application to innercore growth, Geophys. J. Int., 124, 502-524, 1996.

Voller, V. R., A. D. Brent, and C. Prakash, Modelling the mushy region in a binary alloy, Appl. Math. Modelling, 14, 320-326, 1990.

K. Kurita, Earthquake Research Institute, University of Tokyo, Tokyo, Japan.

Y. Ogawa, Earthquake Research Institute, University of Tokyo, 1-1-1 Yoyoi, Bunkyo-ku, Tokyo, Japan. (yoshiko@eri.u-tokyo.ac.jp)

Y. Yamagishi, Institute for Frontier Research on Earth Evolution, Japan Marine Science and Technology Center, Yokosuka, Japan. 